KANSAS JOURNAL of MEDICINE

\section{Annual Report of the University of Kansas Health System Poison Control Center}

Elizabeth Silver, Pharm.D., Lisa K. Oller, Pharm.D.,

Doyle M. Coons, R.N., Stephen L. Thornton, M.D.

Kansas Poison Control Center at the University of Kansas

Health System, Kansas City, KS

Received Dec. 23, 2019; Accepted for publication Feb. 12, 2020; Published online May 21, 2020

\section{ABSTRACT}

Introduction. This is the 2018 Annual Report of the Kansas Poison Control Center at The University of Kansas Health System (KSPCC). The KSPCC serves the state of Kansas 24-hours per day, 365 days a year with certified specialists in poison information and clinical and medical toxicologists.

Methods. All encounters reported to the KSPCC from January l, 2018 through December 31, 2018 were analyzed. Data recorded for each exposure included caller location, age, weight, gender, exposure substance, nature of exposure, route of exposure, interventions, medical outcome, disposition, and location of care.

Results. There were 21,072 total encounters, including 20,031 human exposure cases. Calls were received from every county and hospital in Kansas. Most of the exposures involved females (51.5\%, $\mathrm{n}=10,320)$ and a child less than 19 year of age $(64 \%, \mathrm{n}=12,865)$. Medical outcomes were 24.5\% $(n=4,912)$ no effect, $17.7 \%(n=3,542)$ minor effect, $9.1 \%(n=1,830)$ moderate effect, and $2.4 \%(n=476)$ major effect. Seven deaths were reported in 2018. The number of exposure calls from healthcare facilities and severity of medical outcomes increased in 2018 compared to 2017.

Conclusion. The 2018 KSPCC annual report demonstrated that the center receives calls from the entire state of Kansas totaling over 20,000 human exposures. While pediatric exposures remain the most common encounter, a trend continued of an increasing number of calls from healthcare facilities and for cases with serious outcomes. This report supported the continued value of the KSPCC to both public and acute health care in the state of Kansas.

\section{Kans J Med 2020;13:90-100 \\ INTRODUCTION}

This is the 2018 Annual Report of Kansas Poison Control Center at The University of Kansas Health System (KSPCC). The KSPCC is a 24-hour, 365 day-a-year health care information resource serving the state of Kansas. It was founded in 1982 and is one of the 55 poison control centers certified by the American Association of Poison Control Centers (AAPCC) in the United States. The KSPCC is staffed by nine certified specialists in poison information who are either critical care trained nurses or doctors of pharmacy. There is 24-hour back-up provided by board certified clinical and medical toxicologists.
The KSPCC receives calls from the public, law enforcement, health care professionals, and public health agencies. Encounters may involve an exposed animal or human (Exposure Call) or a request for information with no known exposure (Information Call). The KSPCC follows all cases to make management recommendations, monitor case progress, and document medical outcome. This information is recorded electronically in the Toxicall ${ }^{\oplus}$ data management system and uploaded in near real-time to the National Poison Data System (NPDS).

NPDS is the data warehouse for all the nation's poison control centers. ${ }^{1}$ The average time to upload data for all poison centers is 8.07 $[7.32,12.65]$ (median [25\%,75\%]) minutes, creating a near real-time national exposure database and surveillance system. The KSPCC has the ability to share NPDS real time surveillance with state and local health departments and other regulatory agencies. An analysis and summary of all encounters reported to the KSPCC from January 1, 2018 through December 31, 2018 follows.

\section{METHODS}

All KSPCC encounters recorded electronically in the Toxicall ${ }^{\circledR}$ data management system from January 1, 2018 to December 31, 2018 were analyzed. Cases were first classified as either an exposure or suspected exposure (Human Exposure, Animal Exposure, Non-Exposure Confirmed Cases) or a request for information with no reported exposure (Information Call). Data extracted included: caller location, age, weight, gender, exposure substance, number of follow up calls, nature of exposure (unintentional, recreational, or intentional), exposure scenario, route of exposure (oral, dermal, parenteral), interventions, medical outcome (no effect, minor, moderate, severe or death), disposition (admitted to noncritical care unit, admitted to critical care unit, admitted to psychiatry unit, lost to follow-up, or treated and released) and location of care (non-health care facility or health care facility).

For this analysis a pediatric case was defined as any patient 19 years of age or less. This is consistent with NPDS methodology. Similarly, NPDS descriptions of the medical outcomes of cases were used. Minor outcomes are defined as minimally bothersome symptoms while moderate outcomes are more pronounced symptoms, usually requiring treatment, and major outcomes are life threatening signs and symptoms. Data was analyzed using Microsoft ${ }^{\circledast}$ Excel (Microsoft Corp, Redmond, WA).

\section{RESULTS}

The KSPCC logged 21,072 total calls in 2018, including 20,031 human exposure cases, 74 non-exposure confirmed cases, 92 animal exposure cases, and 875 information calls. This was a decrease of 359 calls (1.7\%) compared to 2017. For information calls, drug information $(\mathrm{n}=285)$ was the most common reason for calling. Table 1 further describes the encounter types. The KSPCC made 30,589 follow-up calls in 2018 . Follow-up calls were done in $54.5 \%$ of human exposure cases. One follow-up call was made in $23.1 \%$ of human exposure cases and multiple follow-up calls (range 2 - 45) were made in $31.4 \%$ of cases. In human exposure calls for which follow-up calls were made, an average of 2.8 calls per case were performed, which was a $5 \%$ increase over 2017. 
Table 1. Encounter type.

\begin{tabular}{|c|c|c|}
\hline & Number & $\%$ \\
\hline \multicolumn{3}{|l|}{ Exposure } \\
\hline Human exposure & 20,031 & 99.5 \\
\hline Animal exposure & 92 & 0.5 \\
\hline Subtotal & 20,123 & 95.5 \\
\hline \multicolumn{3}{|l|}{ Non-Exposure Confirmed Cases } \\
\hline Human non-exposure & 74 & 0.4 \\
\hline Subtotal & 74 & 0.4 \\
\hline \multicolumn{3}{|l|}{ Information Call } \\
\hline Drug information & 285 & 32.6 \\
\hline Drug identification & 94 & 10.7 \\
\hline Environmental information & 97 & 11.1 \\
\hline Medical information & 27 & 3.1 \\
\hline Occupational information & 3 & 0.3 \\
\hline Poison information & 85 & 9.7 \\
\hline Prevention / Safety / Education & 19 & 2.2 \\
\hline Teratogenicity information & 6 & 0.7 \\
\hline Other information & 44 & 5.0 \\
\hline Substance abuse & 7 & 0.8 \\
\hline Administrative & 19 & 2.2 \\
\hline Caller referred & 189 & 21.6 \\
\hline Subtotal & 875 & 4.1 \\
\hline Total & 21,072 & 100.0 \\
\hline
\end{tabular}

The KSPCC received calls from all 105 counties and every hospital in Kansas. The county with the largest number of calls was Sedgwick County with 3,218. In addition, calls were received from 46 states, the District of Columbia, and the U.S. Virgin Islands, while eight calls came from foreign countries including Mexico and Thailand.

Overall, a slight majority of human exposure cases $(51.5 \%, \mathrm{n}=$ 10,320) were female. In children younger than 13 years of age, most encounters involved a male, but this gender distribution was reversed in teenagers and adults. Approximately 64\% $(n=12,865)$ of human exposures involved a child (defined as age 19 years or less). Table 2 illustrates distribution of human exposures by age and gender.

Patients one year of age were the most common age group involved in encounters reported to the KSPCC. For adults, the age group of 20-29 years old was most commonly encountered. Seventy-three exposures occurred in pregnant women (0.4\% of all human exposures). Of these exposures, $31.5 \%$ occurred in the first trimester, $37 \%$ occurred in the second trimester, and $28.8 \%$ occurred in the third trimester. Most of the pregnancy exposures $(n=44,60.3 \%)$ were unintentional exposures, but there were 21 (28.8\%) intentional exposures. There were no reported deaths to KSPCC in pregnant women in 2018.

For human exposures, $67.2 \%(n=13,455)$ of calls originated from a residence (own or other), while $93.5 \%(n=18,731)$ of these exposures occurred at a residence (own or other). Calls from a health care facility accounted for $25.8 \%(n=5,168)$ of human exposure encounters. Table 3 further details the origin of human exposure calls and where the exposure took place.
KANSAS JOURNAL of MEDICINE 2018 KSPCC REPORT

continued.

The majority of human exposures, 85.6 \% $(n=17,150)$, were acute cases defined as exposures occurring over eight hours or less. Chronic exposures (exposures occurring over eight hours) accounted for $2.0 \%$ (404) of all human exposures reported. Acute on chronic exposures (single exposure that was preceded by a chronic exposure over eight hours) totaled 2,382 (11.9\%). Ingestion was the most common route of exposure documented $(81.9 \%, \mathrm{n}=17,554)$ in all cases. Table 4 further details the routes of exposures.

The most common reported substance in those less than six years of age was cosmetics/personal care products $(n=1,134)$ followed closely by household cleaning products $(n=1,125)$. For adult $(>19$ years of age) encounters, analgesics $(n=1,210)$ and sedatives/ hypnotics/antipsychotics $(n=1,147)$ were the most frequently involved substances. Among all encounters, analgesics $(n=2,867,11.6 \%)$ were the most frequently encountered substance category. Table 5 lists most frequently encountered substance categories for pediatric encounters and Table 6 lists those for adult encounters. Appendix A is a summary log for all exposures categorized by category and subcategory of substance (available at journals.ku.edu/kjm).

There was a total of 316 plant exposures reported to the KSPCC. The most common plant exposure encountered was to pokeweed (Phytolacca Americana; $\mathrm{n}=44$ ). Table 7 lists the top five most encountered plants.

Unintentional exposures were the most common reason for exposures $(76.7 \%, \mathrm{n}=15,364)$, while intentional exposures accounted for $20.7 \%(n=4,140)$ of exposures. Table 8 lists reasons for human exposures. A majority of unintentional exposures, $63.5 \%(n=9,759)$, occurred in the $\leq 5$-year-old age group. Up to 12 years of age, $98.2 \%$ $(n=10,830)$ of ingestions were unintentional. However, in the 13 19-year-old group, intentional exposure was most common (69.5\%, $\mathrm{n}=1,277)$. In total, suspected suicide attempts accounted for $15.7 \%$ $(n=3,138)$ of human encounters. When a therapeutic error was the reason for exposure, a double dose was the most common scenario, $30 \%(\mathrm{n}=760)$. Table 8 demonstrates all reasons for human exposures.

Most encounters $(67.4 \%, \mathrm{n}=13,503)$ were managed in a non-health care facility (i.e., a residence). Of the 6,321 encounters managed at a health care facility, $46.1 \%(\mathrm{n}=2,904)$ were admitted. Table 9 lists the management site of all human encounters.

Among human exposures, 15,132 involved exposures to pharmaceutical agents, while 9,510 involved exposure to nonpharmaceuticals. Because an encounter could include numerous pharmaceutical agents and non-pharmaceutical agents, this total is greater than the total number of encounters. However, $86.8 \%$ $(n=17,389)$ of all human exposures were exposed to only a single substance. Among these single substance exposures, the reason for exposure was intentional in $24.5 \%(n=2,188)$ of pharmaceuticalonly cases compared to $3.8 \%(n=323)$ of non-pharmaceutical single substance exposures. 


\section{KANSAS JOURNAL of MEDICINE}

\section{KSPCC REPORT}

continued.

Table 2. Distribution of human exposures by age and gender.

\begin{tabular}{|c|c|c|c|c|c|c|c|c|c|c|}
\hline & \multicolumn{2}{|c|}{ Male } & \multicolumn{2}{|c|}{ Female } & \multicolumn{2}{|c|}{ Unknown Gender } & \multicolumn{2}{|c|}{ Total } & \multicolumn{2}{|c|}{ Cumulative Total } \\
\hline Age (yrs.) & $\mathbf{N}$ & $\begin{array}{c}\% \text { of age } \\
\text { group } \\
\text { total }\end{array}$ & $\mathbf{N}$ & $\begin{array}{c}\% \text { of age } \\
\text { group } \\
\text { total }\end{array}$ & $\mathbf{N}$ & $\begin{array}{c}\% \text { of age } \\
\text { group total }\end{array}$ & $\mathbf{N}$ & $\begin{array}{l}\% \text { of total } \\
\text { exposure }\end{array}$ & $\mathbf{N}$ & $\%$ \\
\hline$<1$ year & 595 & 54.09 & 503 & 45.73 & 2 & 0.18 & 1,100 & 5.49 & 1,100 & 5.49 \\
\hline lyear & 1,674 & 52.81 & 1,494 & 47.13 & 2 & 0.06 & 3,170 & 15.83 & 4,270 & 21.32 \\
\hline 2 years & 1,548 & 51.48 & 1,457 & 48.45 & 2 & 0.07 & 3,007 & 15.01 & 7,277 & 36.33 \\
\hline 3 years & 798 & 56.80 & 607 & 43.20 & 0 & 0.00 & 1,405 & 7.01 & 8,682 & 43.34 \\
\hline 4 years & 400 & 55.56 & 317 & 44.03 & 3 & 0.42 & 720 & 3.59 & 9,402 & 46.94 \\
\hline 5 years & 238 & 60.41 & 156 & 39.59 & 0 & 0.00 & 394 & 1.97 & 9,796 & 48.90 \\
\hline Unknown $\leq 5$ years & 0 & 0.00 & 1 & 100.00 & 0 & 0.00 & 1 & 0.00 & 9,797 & 48.91 \\
\hline Child 6-12 years & 689 & 56.34 & 531 & 43.42 & 3 & 0.25 & 1,223 & 6.11 & 11,020 & 55.01 \\
\hline Teen 13-19 years & 660 & 35.91 & 1,177 & 64.04 & 1 & 0.05 & 1,838 & 9.18 & 12,858 & 64.19 \\
\hline Unknown Child & 1 & 14.29 & 3 & 42.86 & 3 & 42.86 & 7 & 0.03 & 12,865 & 64.23 \\
\hline Subtotal & 6,603 & 51.33 & 6,246 & 48.55 & 16 & 0.12 & 12,865 & 64.23 & 12,865 & 64.23 \\
\hline 20-29 years & 862 & 46.15 & 1,006 & 53.85 & 0 & 0.00 & 1,868 & 9.33 & 14,733 & 73.55 \\
\hline $30-39$ years & 678 & 43.80 & 867 & 56.01 & 3 & 0.19 & 1,548 & 7.73 & 16,281 & 81.28 \\
\hline 40-49 years & 415 & 39.79 & 626 & 60.02 & 2 & 0.19 & 1,043 & 5.21 & 17,324 & 86.49 \\
\hline $50-59$ years & 387 & 40.40 & 570 & 59.50 & 1 & 0.10 & 958 & 4.78 & 18,282 & 91.27 \\
\hline 60-69 years & 343 & 42.77 & 458 & 57.11 & 1 & 0.12 & 802 & 4.00 & 19,084 & 95.27 \\
\hline 70-79 years & 221 & 42.75 & 296 & 57.25 & 0 & 0.00 & 517 & 2.58 & 19,601 & 97.85 \\
\hline $80-89$ years & 88 & 36.97 & 150 & 63.03 & 0 & 0.00 & 238 & 1.19 & 19,839 & 99.04 \\
\hline$\geq 90$ years & 18 & 40.91 & 26 & 59.09 & 0 & 0.00 & 44 & 0.22 & 19,883 & 99.26 \\
\hline Unknown adult & 48 & 41.74 & 67 & 58.26 & 0 & 0.00 & 115 & 0.57 & 19,998 & 99.84 \\
\hline Subtotal & 3,060 & 42.90 & 4,066 & 57.00 & 7 & 0.10 & 7,133 & 35.61 & 19,998 & 99.84 \\
\hline Unknown age & 12 & 36.36 & 8 & 24.24 & 13 & 39.39 & 33 & 0.16 & 20,031 & 100.00 \\
\hline Total* & 9,675 & 48.30 & 10,320 & 51.52 & 36 & 0.18 & 20,031 & 100.00 & 20,031 & 100.00 \\
\hline
\end{tabular}

*Total includes 33 unknown age cases.

Table 3. Origin of call and site of exposure for human exposure cases.

\begin{tabular}{|l|c|c|c|c|}
\hline \multicolumn{1}{|c|}{ Site } & \multicolumn{2}{|c|}{ Origin of Call } & \multicolumn{2}{c|}{ Site of Exposure } \\
\hline & $\mathbf{N}$ & $\%$ & & N \\
\hline Residence & & & 18,064 & 90.18 \\
\hline Own & 13,110 & 65.45 & 667 & 3.33 \\
\hline Other & 345 & 1.72 & 443 & 2.21 \\
\hline Workplace & 307 & 1.53 & 75 & 0.37 \\
\hline Health care facility & 5,168 & 25.80 & 271 & 1.35 \\
\hline School & 38 & 0.19 & 36 & 0.18 \\
\hline Restaurant/food service & 4 & 0.02 & 161 & 0.80 \\
\hline Public area & 75 & 0.37 & 222 & 1.11 \\
\hline Other & 963 & 4.81 & 92 & 0.46 \\
\hline Unknown & 21 & 0.10 & & \\
\hline
\end{tabular}


Table 4. Route of human exposures.

\begin{tabular}{|l|c|c|c|}
\hline & \multicolumn{2}{|c|}{ Human Exposures } \\
\hline Route & $\mathbf{N}$ & \% of All Routes & \% of All Cases \\
\hline Ingestion & 17,554 & 81.94 & 7.63 \\
\hline Dermal & 1,577 & 7.36 & 5.06 \\
\hline Inhalation/nasal & 1,014 & 4.73 & 3.54 \\
\hline Ocular & 709 & 3.31 & 0.95 \\
\hline Bite/sting & 190 & 0.89 & 0.86 \\
\hline Unknown & 172 & 0.80 & 0.78 \\
\hline Parenteral & 156 & 0.73 & 0.08 \\
\hline Other & 17 & 0.08 & 0.06 \\
\hline Otic & 13 & 0.06 & 0.05 \\
\hline Rectal & 10 & 0.05 & 0.02 \\
\hline Aspiration (with ingestion) & 5 & 0.02 & 0.02 \\
\hline Vaginal & 5 & 0.02 & $\mathbf{1 0 6 . 9 4 ^ { * }}$ \\
\hline Total Number of Routes & $\mathbf{2 1 , 4 2 2 ^ { * }}$ & $\mathbf{1 0 0 . 0 0}$ & \\
\hline
\end{tabular}

*Some cases may have multiple routes of exposure documented.

Table 5. Substance categories most frequently involved in exposure for age $\leq 5$ years old.

\begin{tabular}{|l|c|c|c|c|}
\hline Substance Category & All Substance & $\%$ & Single Substance Exposures & $\%$ \\
\hline Cosmetics/Personal Care Products & 1,134 & 11.04 & 1,110 & 11.69 \\
\hline Cleaning substances (Household) & 1,125 & 10.96 & 1,080 & 11.37 \\
\hline Analgesics & 925 & 9.01 & 853 & 8.98 \\
\hline Foreign bodies/toys/miscellaneous & 587 & 5.72 & 575 & 6.05 \\
\hline Antihistamines & 578 & 5.63 & 512 & 5.39 \\
\hline Dietary supplements/herbals/homeopathic & 472 & 4.60 & 437 & 4.60 \\
\hline Vitamins & 451 & 4.39 & 404 & 4.25 \\
\hline Topical preparations & 441 & 4.29 & 434 & 4.57 \\
\hline Pesticides & 386 & 3.76 & 377 & 3.97 \\
\hline Gastrointestinal preparations & 281 & 2.74 & 254 & 2.67 \\
\hline Cold and cough preparations & 226 & 2.20 & 209 & 2.20 \\
\hline Cardiovascular drugs & 222 & 2.16 & 151 & 1.59 \\
\hline Essential oils & 215 & 2.09 & 208 & 2.19 \\
\hline Antimicrobials & 204 & 1.99 & 189 & 1.99 \\
\hline Plants & 201 & 1.96 & 196 & 2.06 \\
\hline
\end{tabular}




\section{KANSAS JOURNAL of MEDICINE}

\section{KSPCC REPORT}

continued.

Table 6. Substance categories most frequently involved in exposures of adults ( $\geq 20$ years).

\begin{tabular}{|c|c|c|c|c|}
\hline Substance Category & All Substance & $\%$ & Single Substance Exposures & $\%$ \\
\hline Analgesics & 1,210 & 11.50 & 559 & 10.45 \\
\hline Sedative/hypnotics/antipsychotics & 1,147 & 10.91 & 315 & 5.89 \\
\hline Antidepressants & 955 & 9.08 & 311 & 5.81 \\
\hline Cardiovascular drugs & 761 & 7.24 & 249 & 4.65 \\
\hline Alcohols & 535 & 5.09 & 67 & 1.25 \\
\hline Antihistamines & 471 & 4.48 & 202 & 3.77 \\
\hline Anticonvulsants & 463 & 4.40 & 142 & 2.65 \\
\hline Cleaning substances (Household) & 445 & 4.23 & 348 & 6.50 \\
\hline Pesticides & 366 & 3.48 & 327 & 6.11 \\
\hline Hormones and hormone antagonists & 328 & 3.12 & 175 & 3.27 \\
\hline Stimulants and street drugs & 303 & 2.88 & 131 & 2.45 \\
\hline Chemicals & 244 & 2.32 & 206 & 3.85 \\
\hline Cosmetics/personal care products & 224 & 2.13 & 198 & 3.70 \\
\hline Fumes/gases/vapors & 212 & 2.02 & 190 & 3.55 \\
\hline Muscle relaxants & 210 & 2.00 & 68 & 1.27 \\
\hline
\end{tabular}

Table 7. Top 5 most frequent plant exposures.

\begin{tabular}{|l|c|}
\hline Botanical Name or Category & N \\
\hline Phytolacca americana (L.) (Botanic name) & 44 \\
\hline Cherry (Species unspecified) & 18 \\
\hline Plants -Toxicodendrol & 16 \\
\hline Philodendron (Species unspecified) & 13 \\
\hline Spathiphyllum species (Botanic name) & 10 \\
\hline Plants-general-unknown & 22 \\
\hline
\end{tabular}


Table 8. Reason for human exposure cases.

\begin{tabular}{|c|c|c|}
\hline \multicolumn{3}{|l|}{ Unintentional } \\
\hline Unintentional - general & 10,383 & 51.8 \\
\hline Unintentional - therapeutic error & 2,446 & 12.2 \\
\hline Unintentional - misuse & 1,381 & 6.9 \\
\hline Unintentional - environmental & 485 & 2.4 \\
\hline Unintentional - occupational & 325 & 1.6 \\
\hline Unintentional - bite/sting & 191 & 1.0 \\
\hline Unintentional - food poisoning & 139 & 0.7 \\
\hline Unintentional - unknown & 14 & 0.1 \\
\hline Subtotal & 15,364 & 76.7 \\
\hline \multicolumn{3}{|l|}{ Intentional } \\
\hline Intentional - suspected suicide & 3,138 & 15.7 \\
\hline Intentional - misuse & 533 & 2.7 \\
\hline Intentional - abuse & 382 & 1.9 \\
\hline Intentional - unknown & 87 & 0.4 \\
\hline Subtotal & 4,140 & 20.7 \\
\hline \multicolumn{3}{|l|}{ Adverse reaction } \\
\hline Adverse reaction - drug & 249 & 1.2 \\
\hline Adverse reaction - other & 56 & 0.3 \\
\hline Adverse reaction - food & 49 & 0.2 \\
\hline Subtotal & 354 & 1.8 \\
\hline \multicolumn{3}{|l|}{ Unknown } \\
\hline Unknown reason & 90 & 0.4 \\
\hline Subtotal & 90 & 0.4 \\
\hline \multicolumn{3}{|l|}{ Other } \\
\hline Other - malicious & 52 & 0.3 \\
\hline Other - withdrawal & 19 & 0.1 \\
\hline Other - contamination/tampering & 12 & 0.1 \\
\hline Subtotal & 83 & 0.4 \\
\hline Total & 20,031 & 100.0 \\
\hline
\end{tabular}

Table 9. Management site of human exposures.

\begin{tabular}{|l|c|c|}
\hline Site of Management & N & $\%$ \\
\hline Managed in healthcare facility & & \\
\hline Treated/evaluated and released & 3,211 & 16.0 \\
\hline Admitted to critical care unit & 1,520 & 7.6 \\
\hline Admitted to noncritical care unit & 826 & 4.1 \\
\hline Admitted to psychiatric facility & 558 & 2.8 \\
\hline Patient lost to follow-up/left AMA & 206 & 1.0 \\
\hline Subtotal (managed in Healthcare facility) & $\mathbf{6 , 3 2 1}$ & $\mathbf{3 1 . 6}$ \\
\hline Managed on site, non-health care facility & 13,503 & 67.4 \\
\hline Other & 15 & 0.1 \\
\hline Refused referral & 177 & 0.9 \\
\hline Unknown & 15 & 0.1 \\
\hline Total & $\mathbf{2 0 , 0 3 1}$ & $\mathbf{1 0 0 . 0}$ \\
\hline
\end{tabular}




\section{KANSAS JOURNAL of MEDICINE}

\section{KSPCC REPORT}

continued.

When medical outcomes were analyzed, 24.5\% $(\mathrm{n}=4,912)$ of human exposures had no effect, $17.7 \%(\mathrm{n}=3,542)$ had minor effect, $9.1 \%(n=1,830)$ had moderate effect, and $2.4 \%(n=476)$ major effect. Moderate effects were more common in the 13 - 19-year-old group, while major effects were more common in those over 20 years of age. Moderate and major effects were most common in those with intentional encounters. More serious outcomes were related to single-substance pharmaceutical exposures, accounting for $42.9 \%$ (n = 3) of the fatalities. Table 10 lists all medical outcomes by age and Table 11 lists outcomes by reason for exposure.
Use of decontamination and specific therapies, including antidotal therapy, is detailed in tables $12 \mathrm{a}$ and $12 \mathrm{~b}$.

There were seven deaths in 2018 reported to the KSPCC. All deaths involved patients 20 years of age or older. Five of the deaths involved intentional exposures. Table 13 details the seven reported deaths.

Table 14 compares key statistics from 2015 to 2018. Total number of calls has declined since 2016. However, number of exposures calls from healthcare facilities and those involving moderate or major outcomes have steadily increased from 2015 to 2018 . The number of reported deaths decreased from 2017 to 2018.

Table 10. Medical outcome of human exposure cases by patient age.

\begin{tabular}{|c|c|c|c|c|c|c|c|c|c|c|c|c|c|c|c|c|}
\hline \multirow[b]{2}{*}{ Outcome } & \multicolumn{2}{|c|}{$\leq 5$ Years } & \multicolumn{2}{|c|}{ 6-12 Years } & \multicolumn{2}{|c|}{ 13-19 Years } & \multicolumn{2}{|c|}{$\geq 20$ Years } & \multicolumn{2}{|c|}{$\begin{array}{l}\text { Unknown } \\
\text { Child }\end{array}$} & \multicolumn{2}{|c|}{$\begin{array}{c}\text { Unknown } \\
\text { Adult }\end{array}$} & \multicolumn{2}{|c|}{$\begin{array}{l}\text { Unknown } \\
\text { Age }\end{array}$} & \multicolumn{2}{|c|}{ Total } \\
\hline & $\mathbf{N}$ & $\%$ & $\mathbf{N}$ & $\%$ & $\mathbf{N}$ & $\%$ & $\mathbf{N}$ & $\%$ & $\mathbf{N}$ & $\%$ & $\mathbf{N}$ & $\%$ & $\mathbf{N}$ & $\%$ & $\mathbf{N}$ & $\%$ \\
\hline No effect & 2,962 & 30.23 & 254 & 20.77 & 478 & 26.01 & 1,203 & 17.14 & 3 & 42.86 & 8 & 6.96 & 4 & 12.1 & 4,912 & 24.52 \\
\hline Minor effect & 1,008 & 10.29 & 238 & 19.46 & 530 & 28.84 & 1,736 & 24.74 & 1 & 14.29 & 25 & 21.74 & 4 & 12.1 & 3,542 & 17.68 \\
\hline Moderate effect & 112 & 1.14 & 52 & 4.25 & 360 & 19.59 & 1,298 & 18.50 & 0 & 0.00 & 3 & 2.61 & 5 & 15.2 & 1,830 & 9.14 \\
\hline Major effect & 14 & 0.14 & 3 & 0.25 & 82 & 4.46 & 375 & 5.34 & 0 & 0.00 & 1 & 0.87 & 1 & 3.0 & 476 & 2.38 \\
\hline Death & 0 & 0.00 & 0 & 0.00 & 0 & 0.00 & 7 & 0.10 & 0 & 0.00 & 0 & 0.00 & 0 & 0.0 & 7 & 0.03 \\
\hline $\begin{array}{l}\text { No follow-up, } \\
\text { nontoxic }\end{array}$ & 341 & 3.48 & 44 & 3.60 & 5 & 0.27 & 39 & 0.56 & 0 & 0.00 & 1 & 0.87 & 0 & 0.0 & 430 & 2.15 \\
\hline $\begin{array}{l}\text { No follow-up, } \\
\text { minimal toxicity }\end{array}$ & 5,032 & 51.36 & 580 & 47.42 & 288 & 15.67 & 1,766 & 25.16 & 3 & 42.86 & 39 & 33.91 & 10 & 30.3 & 7,718 & 38.53 \\
\hline $\begin{array}{l}\text { No follow-up, } \\
\text { potentially toxic }\end{array}$ & 230 & 2.35 & 15 & 1.23 & 55 & 2.99 & 282 & 4.02 & 0 & 0.00 & 29 & 25.22 & 9 & 27.3 & 620 & 3.10 \\
\hline Unrelated effect & 98 & 1.00 & 37 & 3.03 & 40 & 2.18 & 312 & 4.45 & 0 & 0.00 & 9 & 7.83 & 0 & 0.0 & 496 & 2.48 \\
\hline $\begin{array}{l}\text { Death, indirect } \\
\text { report }\end{array}$ & 0 & 0.00 & 0 & 0.00 & 0 & 0.00 & 0 & 0.00 & 0 & 0.00 & 0 & 0.00 & 0 & 0.0 & 0 & 0.00 \\
\hline Total & 9,797 & 100.00 & 1,223 & 100.0 & 1,838 & 100.00 & 7,018 & 100.00 & 7 & 100.00 & 115 & 100.00 & 33 & 100.00 & 20,031 & 100.00 \\
\hline
\end{tabular}

Table 11. Medical outcome by reason for exposure in human exposures.

\begin{tabular}{|c|c|c|c|c|c|c|c|c|c|c|c|c|}
\hline \multirow[b]{2}{*}{ Outcome } & \multicolumn{2}{|c|}{ Unintentional } & \multicolumn{2}{|c|}{ Intentional } & \multicolumn{2}{|c|}{ Other } & \multicolumn{2}{|c|}{ Adverse Reaction } & \multicolumn{2}{|c|}{ Unknown } & \multicolumn{2}{|c|}{ Total } \\
\hline & $\mathbf{N}$ & $\%$ & $\mathbf{N}$ & $\%$ & $\mathbf{N}$ & $\%$ & $\mathbf{N}$ & $\%$ & $\mathbf{N}$ & $\%$ & $\mathbf{N}$ & $\%$ \\
\hline Death & 1 & 0.01 & 5 & 0.12 & 0 & 0.00 & 1 & 0.28 & 0 & 0.00 & 7 & 0.03 \\
\hline Death, indirect report & 0 & 0.00 & 0 & 0.00 & 0 & 0.00 & 0 & 0.00 & 0 & 0.00 & 0 & 0.00 \\
\hline Major effect & 46 & 0.30 & 405 & 9.78 & 7 & 8.43 & 8 & 2.26 & 10 & 11.11 & 476 & 2.38 \\
\hline Minor effect & 2,280 & 14.84 & 1,138 & 27.49 & 16 & 19.28 & 96 & 27.12 & 12 & 13.33 & 3,542 & 17.68 \\
\hline Moderate effect & 540 & 3.51 & 1,194 & 28.84 & 19 & 22.89 & 55 & 15.54 & 22 & 24.44 & 1,830 & 9.14 \\
\hline No effect & 3,900 & 25.38 & 983 & 23.74 & 5 & 6.02 & 13 & 3.67 & 11 & 12.22 & 4,912 & 24.52 \\
\hline No follow-up, nontoxic & 420 & 2.73 & 7 & 0.17 & 0 & 0.00 & 1 & 0.28 & 2 & 2.22 & 430 & 2.15 \\
\hline No follow-up, minimal toxicity & 7,422 & 48.31 & 179 & 4.32 & 17 & 20.48 & 94 & 26.55 & 6 & 6.67 & 7,718 & 38.53 \\
\hline No follow-up, potentially toxic & 424 & 2.76 & 158 & 3.82 & 13 & 15.66 & 12 & 3.39 & 13 & 14.44 & 620 & 3.10 \\
\hline Unrelated effect & 331 & 2.15 & 71 & 1.71 & 6 & 7.23 & 74 & 20.90 & 14 & 15.56 & 496 & 2.48 \\
\hline Total & 15,364 & 100.00 & 4,140 & 100.00 & 83 & 100.00 & 354 & 100.00 & 90 & 100.00 & 20,031 & 100.00 \\
\hline
\end{tabular}


Table 12a. Decontamination provided in human exposures by age.

\begin{tabular}{|l|c|c|c|c|c|c|c|c|}
\hline Decontamination & $\leq \mathbf{5}$ Years & $\mathbf{6 - 1 2}$ Years & 13-19 Years & $\mathbf{2 0}$ Years & Unknown Child & Unknown Adult & Unknown Age & Total \\
\hline Cathartic & 2 & 0 & 2 & 1 & 0 & 0 & 0 & 5 \\
\hline $\begin{array}{l}\text { Charcoal, multiple } \\
\text { doses }\end{array}$ & 3 & 0 & 8 & 8 & 0 & 0 & 0 & 19 \\
\hline Charcoal, single dose & 62 & 11 & 132 & 197 & 0 & 0 & 0 & 402 \\
\hline Dilute/irrigate/wash & 7,404 & 727 & 402 & 2,531 & 2 & 47 & 10 & 11,123 \\
\hline Food/snack & 1,436 & 132 & 85 & 422 & 2 & 5 & 1 & 2,083 \\
\hline Fresh air & 68 & 52 & 32 & 419 & 0 & 19 & 9 & 599 \\
\hline Lavage & 0 & 0 & 0 & 0 & 0 & 0 & 0 & 0 \\
\hline Other emetic & 67 & 6 & 7 & 49 & 0 & 0 & 0 & 129 \\
\hline Whole bowel irrigation & 0 & 0 & 1 & 13 & 0 & 0 & 0 & 14 \\
\hline
\end{tabular}

Table 12b. Therapy provided in human exposures by age.

\begin{tabular}{|c|c|c|c|c|c|c|c|c|}
\hline Therapy & $\leq 5$ Years & 6-12 Years & 13-19 Years & $\geq 20$ Years & Unknown Child & Unknown Adult & Unknown Age & Total \\
\hline \multicolumn{9}{|l|}{ Decontamination } \\
\hline Cathartic & 2 & 0 & 2 & 1 & 0 & 0 & 0 & 5 \\
\hline Charcoal, multiple doses & 3 & 0 & 8 & 8 & 0 & 0 & 0 & 19 \\
\hline Charcoal, single dose & 62 & 11 & 132 & 197 & 0 & 0 & 0 & 402 \\
\hline Dilute/irrigate/wash & 7,404 & 727 & 402 & 2,531 & 2 & 47 & 10 & 11,123 \\
\hline Food/snack & 1,436 & 132 & 85 & 422 & 2 & 5 & 1 & 2,083 \\
\hline Fresh air & 68 & 52 & 32 & 419 & 0 & 19 & 9 & 599 \\
\hline Ipecac & 0 & 0 & 0 & 2 & 0 & 0 & 0 & 2 \\
\hline Other emetic & 67 & 6 & 7 & 49 & 0 & 0 & 0 & 129 \\
\hline Whole bowel irrigation & 0 & 0 & 1 & 13 & 0 & 0 & 0 & 14 \\
\hline \multicolumn{9}{|l|}{ Other Therapies } \\
\hline Alkalinization & 2 & 0 & 47 & 162 & 0 & 0 & 0 & 211 \\
\hline Antiarrhythmic & 0 & 0 & 0 & 3 & 0 & 0 & 0 & 3 \\
\hline Antibiotics & 17 & 9 & 15 & 171 & 0 & 0 & 0 & 212 \\
\hline Anticonvulsants & 0 & 0 & 3 & 2 & 0 & 0 & 0 & 5 \\
\hline Antiemetics & 13 & 11 & 121 & 255 & 0 & 0 & 0 & 400 \\
\hline Antihistamines & 14 & 6 & 15 & 79 & 0 & 1 & 1 & 116 \\
\hline Antihypertensives & 0 & 0 & 0 & 18 & 0 & 0 & 0 & 18 \\
\hline $\begin{array}{l}\text { Antivenom (Immune Fab } \\
\text { fragment) - Not Specified }\end{array}$ & 1 & 3 & 2 & 22 & 0 & 0 & 0 & 28 \\
\hline $\begin{array}{l}\text { Antivenom/antitoxin (Non-Fab) } \\
- \text { Not Specified }\end{array}$ & 0 & 2 & 0 & 8 & 0 & 0 & 0 & 10 \\
\hline Atropine & 4 & 2 & 3 & 9 & 0 & 1 & 0 & 19 \\
\hline Benzodiazepines & 12 & 7 & 109 & 345 & 0 & 0 & 1 & 474 \\
\hline Bronchodilators & 5 & 6 & 7 & 67 & 0 & 1 & 0 & 86 \\
\hline Calcium & 126 & 8 & 10 & 35 & 0 & 0 & 0 & 179 \\
\hline Cardioversion & 0 & 0 & 0 & 1 & 0 & 0 & 0 & 1 \\
\hline Deferoxamine & 0 & 0 & 0 & 1 & 0 & 0 & 0 & 1 \\
\hline Digoxin Immune Fab & 0 & 0 & 0 & 12 & 0 & 0 & 0 & 12 \\
\hline EDTA & 1 & 0 & 0 & 0 & 0 & 0 & 0 & 1 \\
\hline Ethanol & 0 & 0 & 0 & 1 & 0 & 0 & 0 & 1 \\
\hline Extracorp. procedure (other) & 0 & 0 & 0 & 1 & 0 & 0 & 0 & 1 \\
\hline
\end{tabular}




\section{KANSAS JOURNAL of MEDICINE}

2018 KSPCC REPORT

continued.

Table 12b. Therapy provided in human exposures by age. cont.

\begin{tabular}{|c|c|c|c|c|c|c|c|c|}
\hline Therapy & $\leq 5$ Years & 6-12 Years & 13-19 Years & $\geq 20$ Years & Unknown Child & Unknown Adult & Unknown Age & Total \\
\hline Fluids, IV & 53 & 31 & 525 & 1,535 & 0 & 3 & 0 & 2,147 \\
\hline Flumazenil & 2 & 3 & 2 & 35 & 0 & 0 & 0 & 42 \\
\hline Folate & 0 & 0 & 0 & 5 & 0 & 0 & 0 & 5 \\
\hline Fomepizole & 0 & 1 & 1 & 24 & 0 & 0 & 0 & 26 \\
\hline Glucagon & 1 & 0 & 3 & 25 & 0 & 1 & 0 & 30 \\
\hline Glucose, $>5 \%$ & 3 & 0 & 5 & 48 & 0 & 1 & 0 & 57 \\
\hline Hemodialysis & 0 & 0 & 1 & 24 & 0 & 0 & 0 & 25 \\
\hline Hyperbaric oxygen & 0 & 0 & 0 & 4 & 0 & 0 & 0 & 4 \\
\hline Insulin & 0 & 0 & 6 & 22 & 0 & 1 & 0 & 29 \\
\hline Intubation & 3 & 0 & 30 & 205 & 0 & 1 & 0 & 239 \\
\hline Methylene blue & 0 & 0 & 0 & 4 & 0 & 0 & 0 & 4 \\
\hline NAC, IV & 3 & 4 & 76 & 177 & 0 & 0 & 0 & 260 \\
\hline NAC, PO & 0 & 0 & 14 & 24 & 0 & 0 & 0 & 38 \\
\hline Nalmefene & 0 & 0 & 0 & 1 & 0 & 0 & 0 & 1 \\
\hline Naloxone & 10 & 3 & 28 & 139 & 0 & 1 & 0 & 181 \\
\hline Neuromuscular blocker & 0 & 0 & 2 & 13 & 0 & 0 & 0 & 15 \\
\hline Octreotide & 2 & 0 & 0 & 2 & 0 & 0 & 0 & 4 \\
\hline Other & 42 & 27 & 98 & 534 & 0 & 2 & 0 & 703 \\
\hline Oxygen & 9 & 4 & 52 & 435 & 0 & 1 & 4 & 505 \\
\hline Physostigmine & 0 & 0 & 4 & 5 & 0 & 0 & 0 & 9 \\
\hline Phytonadione & 1 & 0 & 7 & 21 & 0 & 0 & 0 & 29 \\
\hline Sedation (other) & 7 & 1 & 33 & 213 & 0 & 0 & 0 & 254 \\
\hline Steroids & 9 & 7 & 8 & 66 & 0 & 1 & 0 & 91 \\
\hline Succimer & 7 & 0 & 0 & 7 & 0 & 0 & 0 & 14 \\
\hline Transplantation & 0 & 0 & 0 & 1 & 0 & 0 & 0 & 1 \\
\hline Vasopressors & 2 & 0 & 8 & 53 & 0 & 1 & 0 & 64 \\
\hline Ventilator & 2 & 0 & 32 & 197 & 0 & 1 & 0 & 232 \\
\hline
\end{tabular}

\section{DISCUSSION}

The 2018 Kansas Poison Control Center at The University of Kansas Health System's statistics are mirroring those seen nationally by the other 54 accredited poison control centers nationwide. In 2018, 2,530,238 encounters were logged by poison control, including 2,099,751 human exposures. ${ }^{1}$ Overall encounters showed a $2.96 \%$ ( $\mathrm{n}=77,175)$ decline from 2017 to 2018 , though healthcare facility human exposure cases decreased by only $0.261 \%$ from 2017 . More serious outcomes (moderate, major or death) continue to increase. Nationwide, the five substance classes most frequently involved in adult exposures were analgesics, cleaning substances (household), cosmetics/personal care products, sedative/hypnotics/antipsychotics, and antidepressants, while the top five most common exposures in children age five years or less were cosmetics/personal care products, household cleaning substances, analgesics, foreign bodies/toys/ miscellaneous, and topical preparations. There were 3,111 exposure related fatalities reported nationwide in 2018.
The KSPCC has served the state of Kansas continually 24 hours a day, 365 days a year for 37 years. By receiving over 20,000 calls per year, the KSPCC continues to be an important resource for emergency medical services, public health agencies, and health care facilities in Kansas. Childhood poisonings, both unintentional and intentional, remain a major focus since calls for patients under 19 years of age account for approximately $2 / 3$ of all exposures. However, more serious hospitalized adult cases are becoming an increasing trend. 
KANSAS JOURNAL of MEDICINE

2018 KSPCC REPORT

continued.

Table 13. Details on deaths and exposure related fatalities.

\begin{tabular}{|c|c|c|c|c|c|c|c|}
\hline Age; Sex & Substances & Substance Rank & Cause Rank & Chronicity & Route & Reason & AAPCC RCF $*$ \\
\hline \multirow[t]{2}{*}{$53 ; \mathrm{F}$} & $\begin{array}{l}\text { Acetaminophen/ } \\
\text { Diphenhydramine }\end{array}$ & 1 & 1 & $\mathrm{~A} / \mathrm{C}$ & Ingestion & Intentional- unknown & 3 \\
\hline & Metaxalone & 2 & 2 & & & & \\
\hline $60 ; \mathrm{F}$ & Theophylline & 1 & 1 & $\mathrm{C}$ & Ingestion & Adverse reaction - drug & 3 \\
\hline \multirow[t]{2}{*}{$34 ; \mathrm{M}$} & $\begin{array}{l}\text { Acetaminpphen/ } \\
\text { Dextromethorphan/Doxylamine }\end{array}$ & 1 & 1 & $\mathrm{~A}$ & Ingestion & Intentional - suicide & 3 \\
\hline & Tramadol & 2 & 2 & & & & \\
\hline \multirow[t]{5}{*}{$39 ; \mathrm{F}$} & Quetiapine & 1 & 1 & $\mathrm{~A}$ & Ingestion & Unknown & 4 \\
\hline & Trazodone & 2 & 2 & & & & \\
\hline & Duloxetine & 3 & 3 & & & & \\
\hline & Potassium Chloride & 4 & 4 & & & & \\
\hline & Drug, unknown & 5 & 5 & & & & \\
\hline $65 ; \mathrm{M}$ & Methamphetamine & 1 & 1 & A & Inhalation & Intentional-Abuse & 3 \\
\hline $59 ; \mathrm{M}$ & Drug, unknown & 1 & 1 & $\mathrm{~A} / \mathrm{C}$ & Ingestion & Intentional - Suicide & 6 \\
\hline \multirow[t]{3}{*}{$69 ; \mathrm{F}$} & Drug, unknown & 1 & 1 & $\mathrm{~A}$ & Unknown & Intentional - Suicide & 6 \\
\hline & Citalopram & 2 & 2 & & & & \\
\hline & Baclofen & 3 & 3 & & & & \\
\hline
\end{tabular}

*American Association of Poison Control Centers Relative Contribution to Fatality

Table 14. 2015 to 2018 comparison of select statistics.

\begin{tabular}{|l|c|c|c|c|}
\hline & $\mathbf{2 0 1 5}$ & $\mathbf{2 0 1 6}$ & $\mathbf{2 0 1 7}$ & $\mathbf{2 0 1 8}$ \\
\hline Total cases & 20,109 & 21,965 & 21,431 & 21,072 \\
\hline Calls from healthcare facility & 4,267 & 4,514 & 4,892 & 5,224 \\
\hline Moderate or major outcomes & 1,688 & 1,971 & 2,170 & 2,340 \\
\hline Deaths & 13 & 15 & 16 & 7 \\
\hline
\end{tabular}

The ongoing importance of the KSPCC is reflected in trends that have seen rates of poisonings and overdoses increase at an alarming rate over the last decade. According to the most current available data, drug poisoning-related hospitalizations in the United States have increased 26\%, ${ }^{2,3}$ The National Center for Health Statistics noted over 67,000 overdose related deaths in 2018. Similarly, the KSPCC consistently has seen an increase in the number of calls from healthcare facilities and cases with moderate or major medical outcomes. Over the last four years, calls from healthcare facilities have increased by $21 \%$. At the same time, calls involving moderate or major outcomes increased by $37 \%$.

Several limitations must be noted when interpreting poison center data. Reporting exposures to the KSPCC is voluntary and the KSPCC is not contacted regarding all poisonings in the state of Kansas. Furthermore, in a majority of cases, there is no objective confirmation of exposure.

\section{CONCLUSIONS}

The 2018 KSPCC annual report demonstrated that the center received over 20,000 human exposures called from the entire state of Kansas. While pediatric exposures remain the most common, there continued to be an increasing trend in the number of calls from healthcare facilities and for cases with serious outcomes. In this regard, the experience of the KSPCC is similar to national data. This report supported the continued value of the KSPCC to both the public and healthcare professionals in the state of Kansas.

\section{ACKNOWLEDGMENTS}

The authors acknowledge the staff of the University of Kansas Health System Poison Control Center: Anne-Marie Banks, Amber Ashworth, Damien Emley, Kathy White, Anita Farris, Mark Stallbaumer, Bobbie Jean Wainscott, and Connor Bowman. 


\section{KANSAS JOURNAL of MEDICINE}

2018 KSPCC REPORT

continued.

\section{REFERENCES}

${ }^{1}$ Gummin DD, Mowry JB, Spyker DA, et. al. 2018 Annual Report of the American Association of Poison Control Centers' National Poison Data System (NPDS): 36th Annual Report. Clin Toxicol 2019; 57(12):1220-1413. PMID: 31752545.

2 US Centers for Disease Control and Prevention. Annual Surveillance Report of Drug-Related Risks and Outcomes: United States, 2017. 2017. https://www.cdc.gov/drugoverdose/pdf/pubs/2017-cdc-drug-surveillancereport.pdf. Accessed February 3, 2020.

3 US Centers for Disease Control and Prevention. Annual Surveillance Report of Drug-Related Risks and Outcomes: United States Surveillance. November 1,2019. https://www.cdc.gov/drugoverdose/pdf/pubs/2019-cdcdrug-surveillance-report.pdf. Accessed December 6, 2019.

4 Ahmad FB, Escobedo LA, Rossen LM, et al. Provisional drug overdose death counts. National Center for Health Statistics. January 15, 2020. https://www.cdc.gov/nchs/nvss/vsrr/drug-overdose-data.htm. Accessed February 3, 2020.

Keywords: drug overdose, poisoning, ingestion, toxicology, antidotes 